\title{
Orientation domain diversity in macaque area $\mathrm{V} 2$
}

This article was published in the following Dove Press journal:

Eye and Brain

24 September 2014

Number of times this article has been viewed

\section{Benjamin M Ramsden' \\ Chou P Hung ${ }^{2}$ \\ Anna Wang Roe ${ }^{3}$}

'Department of Psychological and Brain Sciences, Indiana University, Bloomington, IN, USA; ${ }^{2}$ Department of Neuroscience, Georgetown University Medical Center, Washington, DC, USA; ${ }^{3}$ Department of Psychology, Vanderbilt University, Nashville, TN, USA
Correspondence: Anna Wang Roe Department of Psychology, Vanderbilt University, III 2 I st Avenue South, 30 I Wilson Hall, Nashville, TN 37240, USA $\mathrm{Tel}+\mathrm{I} 615343090$ I

Fax + I 6153438449

Email anna.roe@vanderbilt.edu
Abstract: Single orientation domains in primary (V1) and second (V2) visual cortical areas are known to encode the orientation of visual contours. However, the visual world contains multiple and complex contour types. How do these domains handle such complexity? Using optical imaging methods, we have examined orientation response to two types of contours: real (luminance-defined) and illusory (inferred). We find that, unlike area V1, there are multiple types of orientation domain in V2. These include "real only" domains, "higher-order" domains (which respond to an orientation whether real or illusory), and other domains with nonmatching real/illusory orientation preference. We suggest that this plurality of orientation domains in V2 enables the complexities of local and global contour extraction.

Keywords: primate, visual cortex, V2, functional organization, functional domains, optical imaging

\section{Introduction}

The second visual area of the primate (area V2) exhibits an exquisite modular organization of function that is evident at multiple spatial scales. Mapping studies indicate that neurons selective for color, form, or disparity are preferentially organized into millimeter-sized stripe compartments (thin, pale, thick) and that these stripes are further organized into preferentially tuned submillimeter-sized functional clusters or "domains"1-12 (Levitt et $\mathrm{al}^{13}$ and Yoshioka and Dow ${ }^{14}$ ). Responses to oriented stimuli, in particular, are organized into large orientation columns, primarily in the pale and thick stripes of V2. ${ }^{6,7,9,11,15}$

The fact that there are orientation domains in both V1 and V2 begs the question as to what differentiates them. It is known that, in addition to simple orientation features (eg, lines or gratings), V2 of the macaque monkey is also characterized by more complex orientation responses. At least one-third of oriented neurons in macaque V2 are reported to also respond to inferred contours presented at the same orientation. ${ }^{16-18}$ Such inferred contours include contours formed by gap completion, ${ }^{17}$ boundaries inferred by texture or disparity cues, ${ }^{19,20}$ and illusory contours cued by offset abutting line gratings. ${ }^{18,21-24}$ Based on these data, it has been suggested that area V2 may play a key role in the earliest representation of inferred "higher-order" contours. ${ }^{16} \mathrm{We}$ and others have shown that the orientation domains in V2 exhibit similar response to simple contours and higher-order contours (abutting line contours, ${ }^{23,25,26}$ Pan et al). ${ }^{27}$ These data suggest that orientation domains in V2 represent an important stage for calculating "orientation" regardless of cues used to generate generalized orientation percepts. How can such invariance be achieved? How is orientation response to different types 
of cues calculated? Are there different levels of orientation generalization? We hypothesize that orientation domains in V2 are actually quite complex and may be composed of different subdomains. These subdomains could represent orientation response specific to different types of cues. Furthermore, we hypothesize a hierarchy of cue-specific orientation response and higher-order cue-invariant orientation response.

As an initial investigation into this possibility, we have used intrinsic signal optical imaging to probe possible complexity of orientation response in V2. We have chosen to map response to illusory contours of the offset abutting grating type $^{21}$ (Figure 1A, left) because response to this type of contour has been well-characterized and because it is amenable to the optical imaging technique. We find that, consistent with our previous findings, V2 indeed contains a higherorder orientation organization; that is, higher-order contour cells have similar orientation tuning for simple and inferred contours (in this paper, referred to as "real" and "illusory" contours, respectively). However, we also find that orientation domain organization is more complex than predicted. Based on these data, we hypothesize an organizational hierarchy for orientation response in V2.

\section{Results}

In a previous publication ${ }^{23}$ we reported that the relationship between real and illusory response in V1 is "anticorrelated". That is, domains that were activated by real contours were relatively suppressed by similarly oriented illusory contours. We also exhibited an example of a "correlated" relationship between real and illusory response in V2 and suggested the presence of higher-order contour domains in V2. In this paper we present additional examples of $\mathrm{V} 2$ response and focus on the observed real/illusory correlations in $\mathrm{V} 2$. We now present five cases (obtained from five hemispheres of five normal adult macaque monkeys) that show that there is a range of orientation domain types in V2. Stimuli and optical imaging acquisition methods used were identical to those presented in previous reports. ${ }^{23}$ All images are displayed with the lunate sulcus at the top edge just out of field of view. As will be seen from the results, we find there is a good degree of variability between cases. We therefore present the data case by case, as we felt this is the best way to convey the variability.

\section{Mapping illusory contour orientation preference domains in area V2: Case I}

Using the intrinsic optical imaging method, we examined the spatial distribution of illusory contour response in macaque area V2. Figure 1 shows data from Case 1, and exemplifies the procedures used in all cases. We imaged a portion of $\mathrm{V} 2$ directly anterior to V1 at the lip of the lunate sulcus (Figure 1B, left and middle panels). Since most of V2 is buried within the lunate sulcus, typically a $0.5-2-\mathrm{mm}$-wide strip was available on the surface for imaging. To ensure that we were recording from area V2, we included adjacent area V1 within our acquisition field of view and determined the functional V1/V2 border via ocular dominance mapping (Figure 1B, right panel). In this case, the locations of thin, thick, and pale stripe compartments of V2 were determined by anatomical methods (cytochrome oxidase histology, Figure 1C). In other cases, pale and thick stripe locations were determined by the magnitude of imaged orientation response. Thin stripes were mapped either by inference from pale/thick locations (orientation activation is weakest in thin stripes) or by preferential color versus luminance response (Figure 2A, color activation is strongest in the thin stripes).

To map real and illusory response, we designed real and illusory gratings with identical contour spacings. We have previously shown that in $\mathrm{V} 1$, orientation-specific responses to these illusory gratings are independent of inducer orientation or pattern position or presence of line ends ${ }^{23}$ or local luminance fluctuations. ${ }^{28}$ Thus, V1 responses to this illusory grating were not due to local features of this stimulus, but due to the illusory orientation content of the stimulus, a feature known to be encoded by V2 cells.

To investigate illusory contour organization in V2, we collected cortical responses to visual presentation of drifting abutting line gratings of horizontal and vertical orientations (illusory stimulus conditions, Figure 1A, left). "Illusory" orientation maps were obtained by subtracting responses to vertical illusory stimulus conditions from horizontal illusory conditions. Spacings of horizontal and vertical illusory contours were identical to those of real line stimuli. Our intention here was to minimize the possible confounding contributions arising from the small oblique real line inducer elements (common to both stimulus conditions, and thus cancelled out via image subtraction), while maximizing the contributions associated with the illusory contour orientation (different in each stimulus condition, and not cancelled out by image subtraction). Size, orientation, and motion of inducer elements were identical in the two illusory stimulus conditions. If there was no spatial organization for illusory contour orientation, we expected no net difference after image subtraction (ie, a subtraction map that is neutrally gray). Because of the relatively weak illusory signal size, we collected $80-140$ trials to obtain a reasonable signal-to-noise ratio. Thus, to maximize number 
A

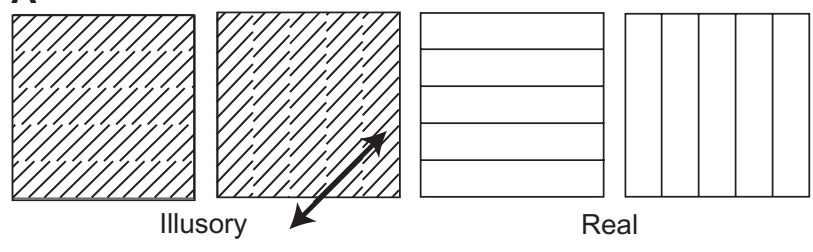

B

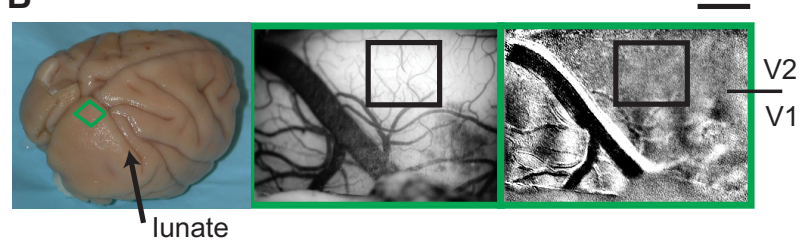

C

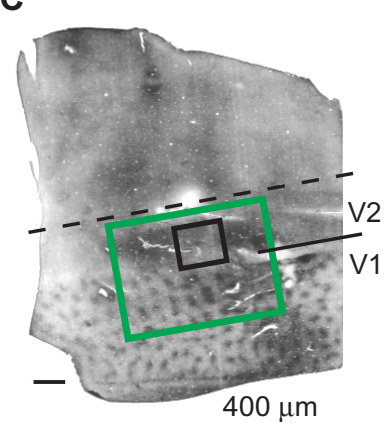

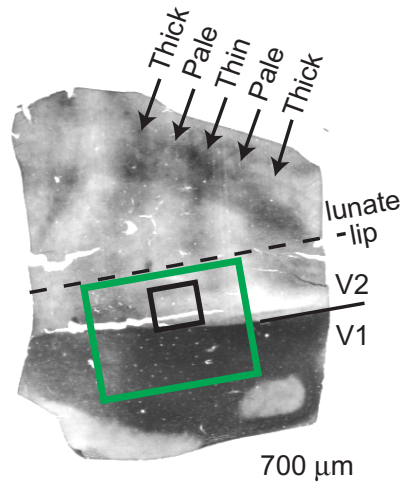

D

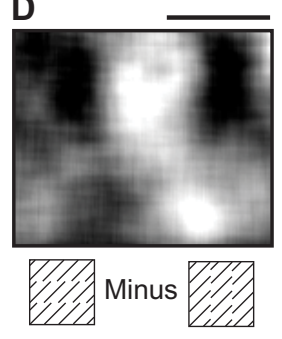

E

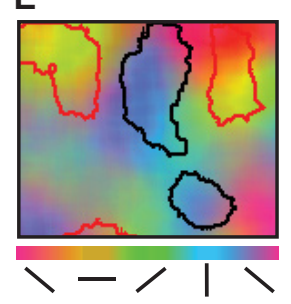

F

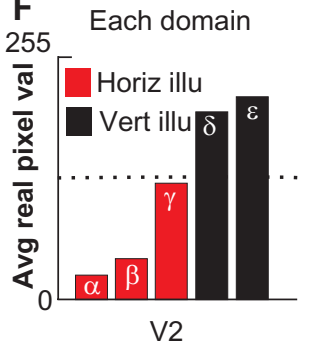

IIIU

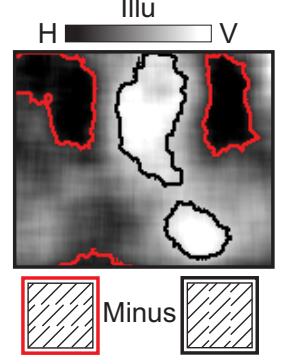

Real

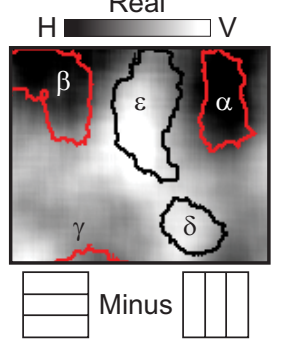

Summed domains

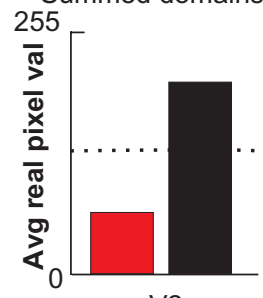

Figure I Functional mapping of illusory and real contour representation in area V2 (Case I).

Notes: (A) Horizontal and vertical illusory (left) and real (right) stimuli were of matched spacing and global luminance, drifting continuously and bidirectionally along the same oblique axis of motion (arrow). Illusory stimuli comprised identical but differently arranged oblique inducer lines. Stimulus portions shown are diagrammatic representations. Actual stimuli were white lines on black backgrounds fully occupying a computer screen. (B) Perfused macaque brain indicating typical optical imaging site (left panel). Imaged areas (green rectangle; 3-5 mm breadth, immediately posterior to lunate sulcus and 5-10 mm lateral to midline) incorporated the functional VI/V2 border with $0.5-2 \mathrm{~mm}$ anteromedial strip of area V2 exposed. Area VI/V2 border was defined by ocular dominance functional mapping (surface vasculature landmarks [middle panel]; termination of dominance columns [right panel]; darkest areas, strongest right eye response; lightest areas, strongest left eye response). Dark oblique band in map is blood vessel artefact. Detailed contour orientation maps were obtained for a portion of V2 (black rectangle). Scale bar I mm. (C) Cytochrome oxidase histology (tangential $30 \mu \mathrm{m}$ slices) anatomically confirmed VI/V2 border location (termination of blob zone, left panel; termination of dark zone, right panel) and thin, pale, and thick stripe compartments of area V2 (arrows, right panel). Imaged zones (green and black rectangles, refer to [B]) were determined using alignments of lesions and vasculature. Scale bar I mm. (D) Functional maps showing imaged illusory orientation domains in area V2 (same case as shown in Ramsden et al ${ }^{23}$ but with new thresholding and analysis methods). Left panel; subtraction of vertical and horizontal illusory contour responses for imaged area (black rectangle [C]). Darkest areas; horizontal illusory contour preference. Lightest areas; vertical illusory contour preference. Right panel; same as left panel but illusory orientation domains demarcated via amplitude thresholding. Horizontal illusory domains, red outlines; vertical illusory domains, black outlines. Grayscale: $-0.01 \%$ to $0.01 \%$ dR/R. Scale bar I mm. (E) Real and illusory orientation domains showed spatial correspondences in V2. Left panel; real orientation map obtained in response to presentations of horizontal, $45^{\circ}$, vertical, and $135^{\circ}$ line gratings. Color shading indicates preferred orientation (net response "vector" to all stimulus orientations). Thresholded illusory domains (red and black outlines from [D]) are superimposed. Vertical real domains (blue) can correspond with vertical illusory domains (black outline). Horizontal real domains (yellow/orange) can correspond with horizontal illusory domains (red outline). Not all domains show strong common real and illusory tuning. Right panel; real orientation difference map obtained in response to subtraction of horizontal and vertical responses. Darkest shading indicates preference for horizontal, lightest shading indicates preference for vertical. Thresholded illusory domains (red and black outlines from [D]) are superimposed, confirming relationships seen in vector map, left panel. Grayscale: $-0.02 \%$ to $0.02 \% \mathrm{dR} / \mathrm{R}$. (F) Spatial correspondence of real/illusory domains confirmed by quantitative pixel analysis. Left panel; average (real) pixel values for each illusory domain in right panel of (E). Pixel values range from 0 (darkest) to 255 (lightest). Horizontal illusory domains (red histogram bars) show lower pixel values than vertical illusory domains (black bars). Right panel; average (real) pixel values across all horizontal or vertical illusory domains.

Abbreviations: dR/R, \% change in reflectance; H, horizontal; V, vertical; Avg, average; Pix, pixel; Val, value; Horiz illu, horizontal illusory; Vert illu, vertical illusory; Illu, Illusory.

of trials (and thereby maximize the signal-to-noise ratio), we collected only two illusory orientations in a single experiment. Due to small signal size, single-condition illusory maps were very difficult to obtain.

Figure 1D (left panel) illustrates an example of a horizontal minus vertical illusory contour grating subtraction. Darkest areas indicate preference for horizontal illusory contours, lightest areas indicate preference for vertical illusory contours, and gray areas indicate equal activation to both stimuli. This case revealed a clustering in activation suggestive of an orientation preference organization. We applied a thresholding algorithm to delineate these illusory orientation preference domains (Figure 1D, right panel; horizontal illusory domains [red outline], vertical illusory 

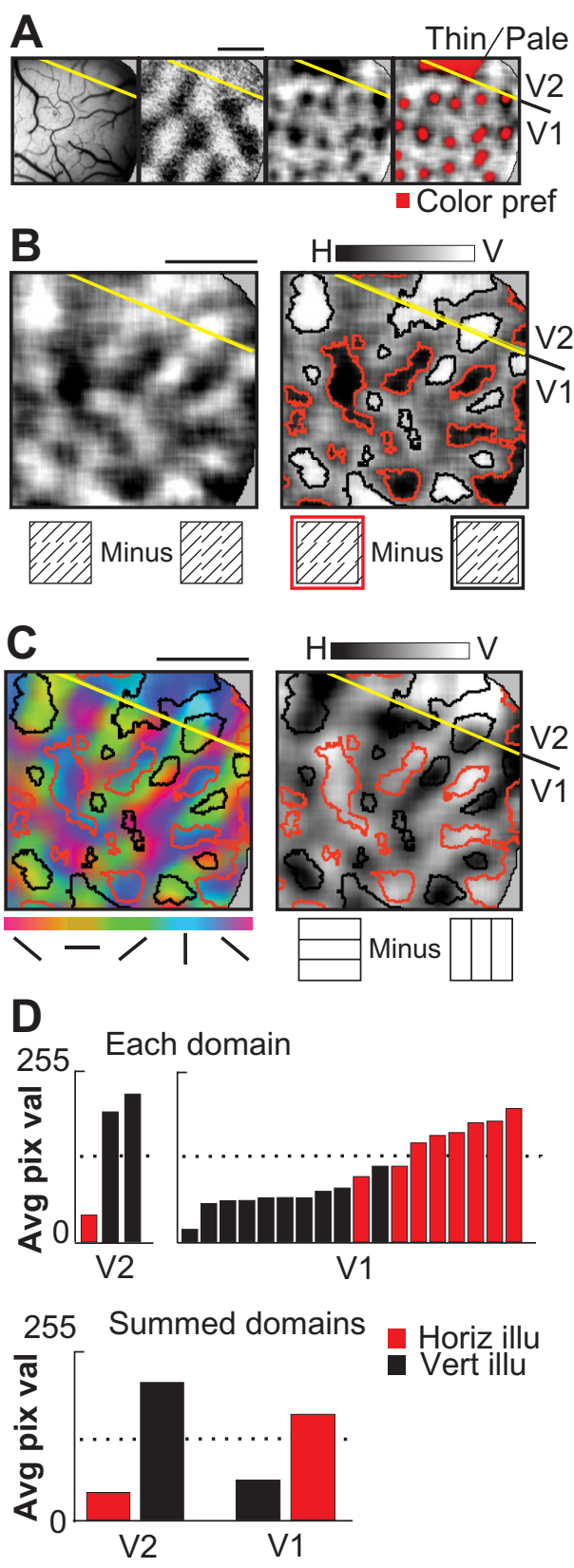

Figure 2 Illusory and real contour representation in macaque areas VI and V2 (Case 2).

Notes: (A) Vessel pattern (left panel) and ocular dominance preference map (second panel) indicating the functional VI/V2 border (yellow line). A small portion of V2 is available for imaging. Third and fourth panels; difference maps for chromatic versus achromatic grating presentations. Dark patches indicate domains preferring chromatic gratings (third panel), highlighted in red (fourth panel). Note blob-like pattern in VI, color stripe compartment in V2. Grey shaded regions at right perimeter indicate portions of signal-obscuring dura. Area imaged is identical for (A-C); scale bars I mm. (B) Larger image of illusory difference map (left panel); horizontal versus vertical illusory contour conditions. Dark and light zones indicate preference for horizontal and vertical illusory contours, respectively. Illusory orientation preference domains are evident in both areas VI and V2. Right panel; thresholded map, highlighting horizontal (red outline) and vertical (black outline) illusory preference domains. Grayscale: -0.0I\% to $0.01 \% \mathrm{dR} / \mathrm{R}$. (C) Real orientation preference map (left panel, vector map, color-coded orientation legend, below). Thresholded illusory orientation preference domains from (B) are superimposed. Vertical illusory domains (black outlines) tend to align with domains preferring vertical real orientations in V2 (blue shading), but tend to align with domains preferring horizontal real orientations in VI (yellow shading). Right panel; difference map (horizontal minus vertical) showing overall relationships are similar to left panel. Dark and light zones indicate preference for horizontal and vertical real orientation, respectively. (D) Different spatial correspondence of real/illusory domains in $\mathrm{VI}$ and $\mathrm{V} 2$ confirmed by quantitative pixel analysis. Top panel; average (real) pixel values for each illusory domain shown in right panel of (C). Pixel values range from 0 (darkest) to 255 (lightest). In V2, vertical illusory domains (black bars) show highest (vertical real) pixel values, while in VI vertical illusory domains show lowest (horizontal real) pixel values. Bottom panel; average (real) pixel values averaged across all horizontal or vertical illusory domains in VI and V2. Grayscale: $-0.02 \%$ to $0.02 \% \mathrm{dR} / \mathrm{R}$. Pixel values range from 0 (darkest) to 255 (lightest). In V2, vertical illusory domains (black bars) show highest pixel values, while horizontal illusory domains (red bars) show lowest real pixel values. Above right panel; average (real) pixel values for each illusory domain in VI shown in right panel of (C). VI reveals opposite relationship between real and illusory domains. Below; average (real) pixel values for all horizontal or vertical illusory domains in V2 (left) and VI (right). In V2, vertical illusory domains (black bars) show highest pixel values, while horizontal illusory domains (red bars) show lowest real pixel values. In VI, vertical illusory domains (black bars) show lowest pixel values, while horizontal illusory domains (red bars) show highest real pixel values.

Abbreviations: dR/R, \% change in reflectance; H, horizontal; V, vertical; Pref, preferred; Avg, average; Pix, pixel; Val, value; Horiz illu, horizontal illusory; Vert illu, vertical illusory. 
domains [black outline]). These thresholded domains ranged from approximately circular to somewhat elliptical in shape and ranged from approximately $0.25 \mathrm{~mm}$ to more than 0.75 $\mathrm{mm}$ in size. To ensure that these activations were not simply artefacts associated with underlying blood vessel distribution, we also compared their distribution with the associated cortical surface blood vessel pattern (Figure 1B, middle panel). Using cytochrome oxidase histology, we determined that this imaged cortex was located within a V2 pale stripe (Figure 1C), a V2 compartment known to show strong orientation preference for real oriented lines and gratings. This initial map suggested that there is "structure" in the illusory contour functional map in area V2 cortex of the anesthetized monkey.

"Real" orientation maps were also obtained in response to drifting horizontal, $45^{\circ}$, vertical, and $135^{\circ}$ line gratings (real stimulus conditions, Figure $1 \mathrm{~A}$, right). Color-coded orientation maps were derived by pixel-wise vector summation of response to the four orientations. As shown in Figure 1E (left panel), horizontal is encoded in yellow, $45^{\circ}$ in green, vertical in blue, and $135^{\circ}$ in red; magnitude of response is indicated by color saturation. Consistent with previous reports,,${ }^{9,23}$ orientation domains in V2 were typically larger than those in V1 (about $500 \mu \mathrm{m}$ in size in V2).

To assess the relationship between illusory and real orientation response, the thresholded illusory domains delineated in Figure 1D (right panel) were then superimposed over the color-coded real orientation vector map (Figure 1E, left panel). Thresholds were computed from the maps obtained by averaging multiple trials of each condition. As we previously reported, ${ }^{23}$ in this case we observed a "like-with-like" correspondence between real and illusory orientation domains. Horizontal illusory orientation domains (red outlines) tended to coincide with horizontal real orientation preference (yellow/orange color code, Figure 1E, left) rather than other orientation preference. Similarly, vertical illusory orientation domains (black outlines) tended to coincide with vertical real orientation preference (blue color code, Figure 1E, left).

To rule out the possibility that these domain associations were spurious artefacts associated with the derivation of our color-coded real vector map, we performed a further map comparison. Grayscale difference maps were obtained by subtracting vertical from horizontal real orientation responses (Figure 1E, right). This produced a map qualitatively similar, though not identical, to the illusory orientation map (compare Figure 1D right panel with Figure 1E right panel). Red outlined horizontal illusory domains were associated with darkly shaded horizontal real domains; black outlined vertical illusory domains were associated with lightly shaded vertical real domains. Note that there are regions of illusory response that have relatively weak real response (eg, area around small red outline), an aspect that will be discussed further in subsequent examples.

We quantified these observations by calculating average (real map) pixel values within each demarcated illusory domain. Figure 1F graphically tabulates average pixel values for horizontal and vertical illusory domains. Low average pixel values (dark in maps, horizontal real preference) tended to be associated with horizontal (red outline) illusory domains. Conversely high average pixel values (light in maps, vertical real preference) tended to be associated with vertical (black outline) illusory domains.

Thus, in Case 1, the aligned correspondence of the real and illusory maps is consistent with our initial prediction derived from single-unit studies. This finding suggested the presence of higher-order contour domains in V2 which encode a particular orientation regardless of whether the contour is real or illusory. ${ }^{23}$ To examine this possible relationship further, we obtained real and illusory maps in four other monkeys.

\section{Case 2}

Figure 2 shows V1/V2 cortex images (Case 2) where only a small portion of $\mathrm{V} 2$ cortex is visible within our acquisition field of view (Figure 2A, left panel; lunate sulcus is just out of view, at the top of the figure border). The location of the V1/V2 border is evident from the extent and abrupt termination of the ocular dominance columns which functionally define area V1 within this field of view (Figure 2A, second panel). Subtraction of achromatic grating responses from isoluminant chromatic grating responses revealed activation domains sensitive to color: blob-like activation in V1 and a larger dark activation in V2 indicating the location of a thin stripe (Figure 2A, third and fourth panel).

An illusory contour subtraction map (Figure 2B, left panel) was obtained, revealing structure within both V1 and V2. Thresholded illusory contour domains (Figure 2B, right panel) are shown overlaid onto the real orientation preference maps (vector orientation map, Figure 2C, left panel; difference map, Figure 2C, right panel). In addition to functional organization within area V2, these data indicate that area V1 also exhibits organized orientation response during illusory contour stimulation. Previously, it has been shown that V1 domains preferring a particular real orientation also paradoxically 
A

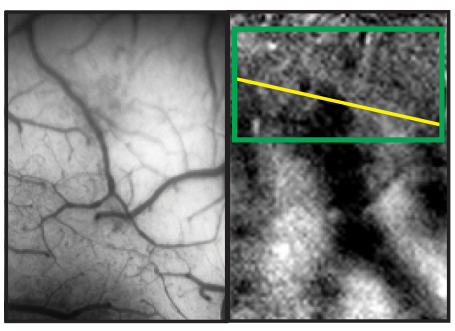

B
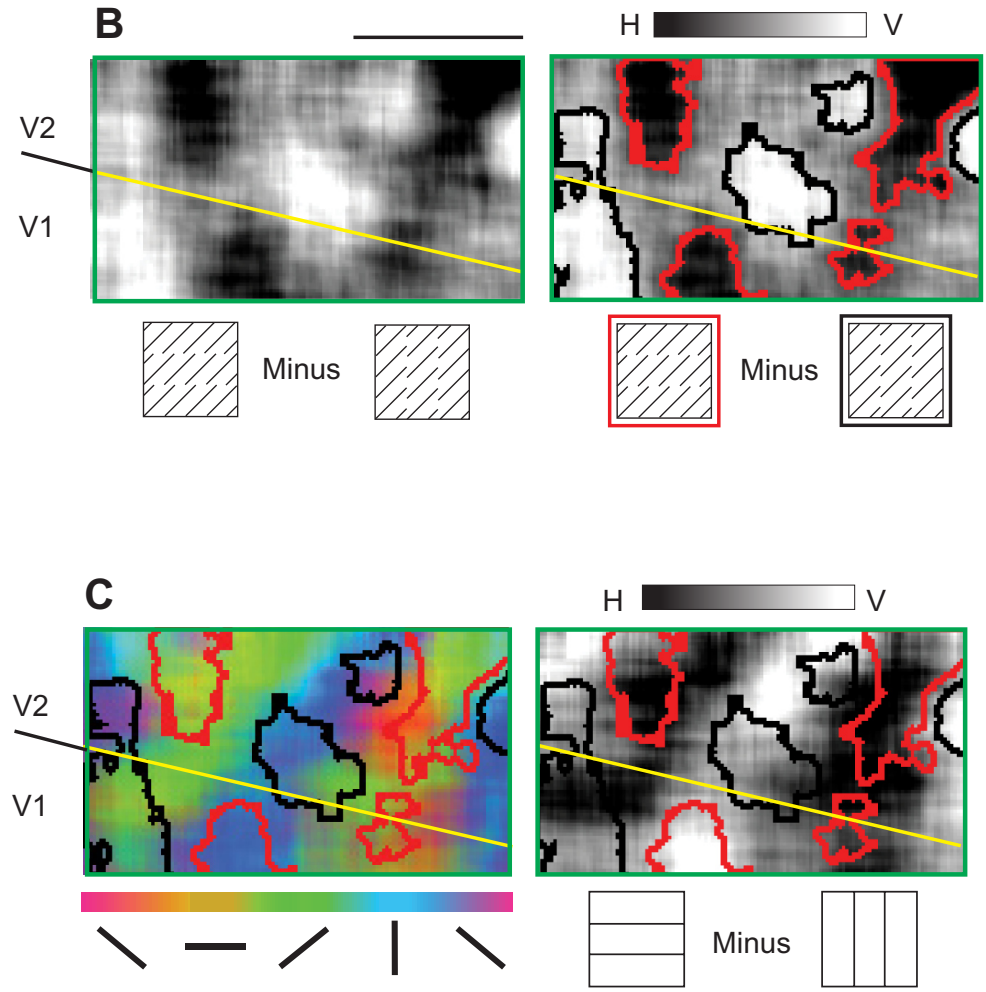

D

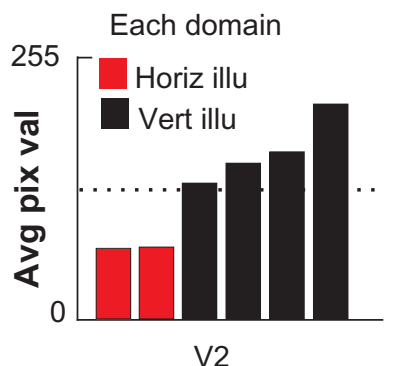

Summed domains

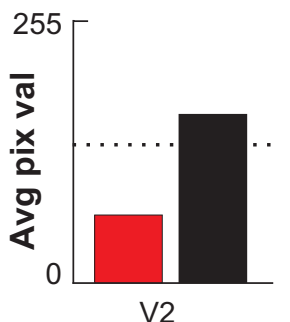

Figure 3 Illusory and real contour representation in macaque areas VI and V2 (Case 3).

Notes: (A) Vessel pattern (left panel) and ocular dominance preference map (right panel) indicating the functional VI/V2 border (yellow line). Approximately I mm of V2 (anteroposterior dimension) is available for imaging. Green rectangle indicates portion of imaged V2 area shown in (B and C). Scale bar I mm. (B) Illusory subtraction map; horizontal versus vertical illusory contour conditions. Dark and light zones indicate preference for horizontal and vertical illusory contours, respectively. Illusory orientation preference domains are evident in area V2. Right panel; thresholded map, highlighting horizontal (red outline) and vertical (black outline) illusory preference domains. Grayscale: $-0.01 \%$ to $0.01 \% \mathrm{dR} / \mathrm{R}$. (C) Real orientation preference maps. Left panel; vector map, color-coded orientation legend, below. Thresholded illusory orientation preference domains from (B) are superimposed. In V2, vertical illusory domains (black outlines) tend to align with vertical real domains (blue shading). But in VI, the horizontal illusory domain (red outline) aligned with the vertical real domain. Right panel; difference map, horizontal minus vertical responses. The overlap relationships are similar to those shown in vector map. Grayscale: $-0.02 \%$ to $0.02 \% \mathrm{dR} / \mathrm{R}$. Scale bar I $\mathrm{mm}$. (D) Real/illusory domain correspondence in V2 confirmed by quantitative pixel analysis. Left panel; average (real) pixel values for each illusory domain shown in right panel of (C). Pixel values range from 0 (darkest) to 255 (lightest). In V2, vertical illusory domains (black bars) show highest pixel values, while horizontal illusory domains (red bars) show lowest real pixel values. Right panel; average (real) pixel values for all horizontal or vertical illusory domain shown. Pixel values range from 0 (darkest) to 255 (lightest). In V2, vertical illusory domains (black bars) show highest pixel values, while horizontal illusory domains (red bars) show lowest real pixel values.

Abbreviations: $\mathrm{dR} / \mathrm{R}$, \% change in reflectance; $\mathrm{H}$, horizontal; $\mathrm{V}$, vertical; Avg, average; Pix, pixel; Val, value; Horiz illu, horizontal illusory; Vert illu, vertical illusory. 
prefer the orthogonal (rather than matching) illusory contour orientation. ${ }^{23}$ Thus, in V1 of Figure $2 \mathrm{C}$ horizontal illusory domains (red outlines) coincide with vertical real domains (blue color code), while vertical illusory domains (black outlines) coincide with horizontal real domains (yellow/orange color code). This paradoxical anticorrelated or "inverted" V1 domain relationship, however, is not observed in V2 of this case. Rather, in V2, the thresholded illusory domains tended to show a matched orientation preference for real and illusory oriented stimulus conditions (eg, black outlined domains indicating vertical illusory preference are associated with blue color code indicating real vertical preference, Figure $2 \mathrm{C}$, left panel). The relative alignments between real and illusory domains were especially striking across the V1/V2 border in this case (note the change in color-coding V1 within the black outlined domains, Figure 2C, left panel; from blue [vertical] in V2 to yellow [horizontal] in V1). These like-with-like associations in V2 were confirmed when illusory domain alignments were compared with the grayscale difference map conditions (eg, black outlined domains coincide with lightest shadings, Figure 2C, right panel).

The observed relationship between real and illusory domains was quantitatively confirmed by calculating average pixel values within each illusory domain (Figure 2D). As with Case 1, in V2 (graphs on left) low average pixel values (dark, indicating horizontal real preference) tended to be associated with horizontal illusory domains (red bars). Conversely high average pixel values (light, indicating vertical real preference) tended to be associated with vertical illusory domains (black bars). In contrast, in V1, the opposite relationship was observed: low average pixel values (horizontal real) were associated with vertical illusory (black bars) and high average pixel values (vertical real) were associated with horizontal illusory (red bars). This illustrates that the current quantitative methods reveal an anticorrelated relationship in V1, similar to that reported previously with different methods. ${ }^{23}$ Since this V1 relationship has been extensively documented, we focus on V2 in subsequent examples.

Von der Heydt and Peterhans ${ }^{18}$ reported that less than half of orientation-selective cells in V2 are also tuned to offset abutting line grating illusory contours. This predicts that there should be some orientation-selective cells in V2 that are not responsive to illusory contours. Consistent with this prediction, we find some real orientation preference regions in V2 which were unresponsive to illusory orientation (eg, the light zone between the two black outlined vertical illusory domains in V2, Figure $2 \mathrm{C}$, right panel). Thus, although we find a coalignment of illusory orientation domains with real orientation domains, this does not mean that all regions responsive to (vertical) real are also responsive to (vertical) illusory. In sum, although there is only a small area of $\mathrm{V} 2$ visible in this field of view, the domains available are consistent with the presence of contour domains activated by both real and illusory stimuli.

\section{Case 3}

In a third case (Figure 3), we focused on a region in V2 with strong real orientation response, presumably a pale/thick stripe region. Again, the subtraction of horizontal and vertical illusory images revealed distinct illusory orientation preference domains in V2 (Figure 3B, left; thresholded data, Figure $3 \mathrm{~B}$, right). When illusory map outlines were overlaid on real orientation maps (Figure 3C), again we observed an association between vertical illusory and vertical real domains in V2 (black outlines predominantly associated with blue regions, Figure 3C, left panel; black outlines predominantly associated with lighter regions, Figure $3 \mathrm{C}$, right panel) and horizontal illusory domains tended to align with horizontal orientation preference regions (red outlines tended to overlap with yellow regions, Figure 3C, left panel; red outlines tended to overlap with darker shadings; Figure 3C, right panel). As in Case 2, note that the reversal of these real/illusory associations in $\mathrm{V} 1$ (eg, near the V1/V2 border in Figure 3C, the association of the leftmost black outline changes from blue/purple in V2 to yellow/green in V1; the red outline in V1 is associated with blue rather than yellow). These relationships are quantified in Figure 3D. When pixel values from the real difference map were averaged and tabulated for each illusory domain, V2 exhibited a coaligned horizontal real/illusory (red outline with low pixel values) and vertical real/illusory (black outline with high pixel values), and V1 exhibited an inverse relationship (red outline with high pixel values and black outline with low pixel values).

As noted for previous cases, there were also some real horizontal (dark pixels) and vertical (light pixels) response regions in V2 which were not associated with either horizontal or vertical illusory response domains (Figure 3C). Similarly, some parts of the illusory domains (eg, small black outlined domain closest to the top in Figure 3C) overlaid real response to a slightly different orientation. These data thus suggested some association between real and illusory regions of different orientation preferences. To further examine this association, we asked whether this reflects inducer orientation response (green pixels in this case). Although the horizontal minus vertical illusory subtraction removes the inducer component (which is common to both stimuli, see Figure 11 
A

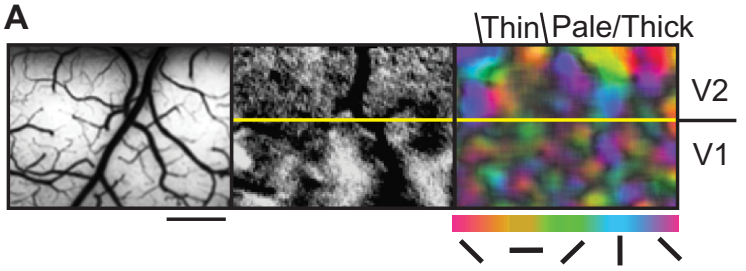

B Thin Pale/Thick
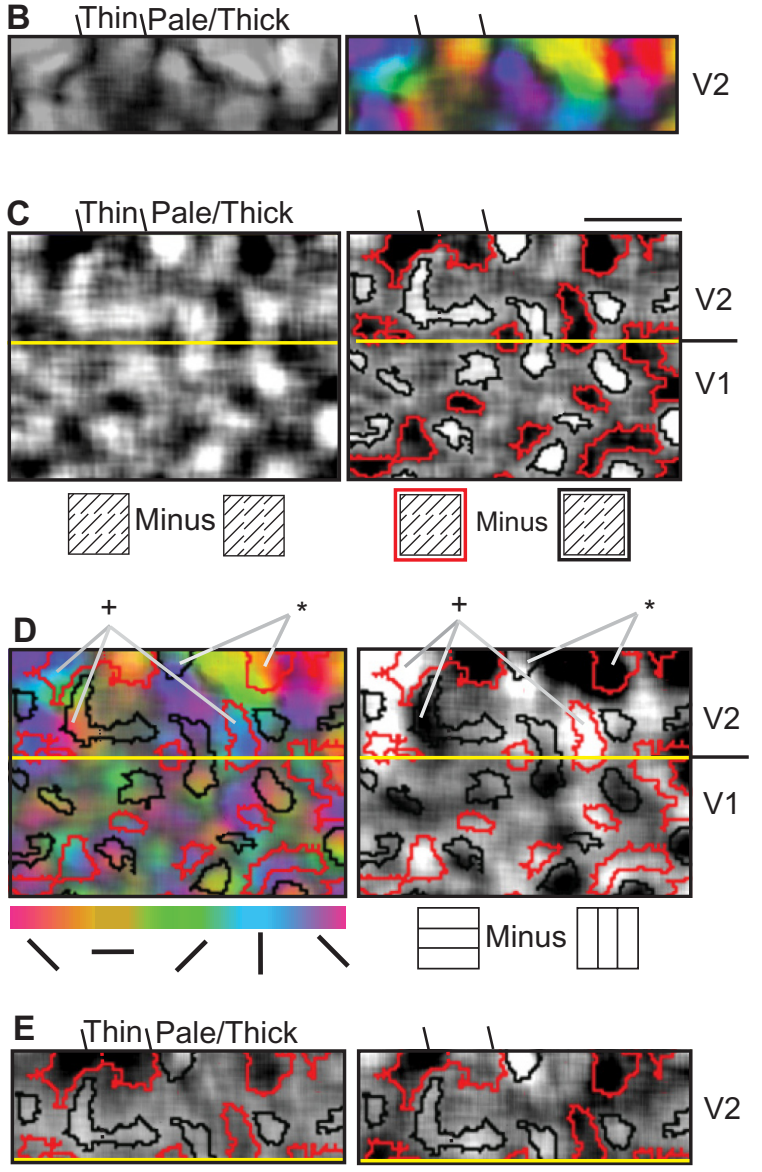

F Each domain

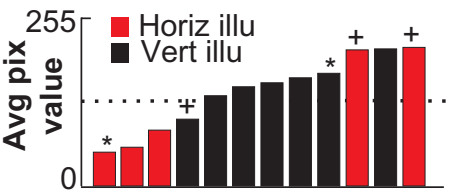

V2

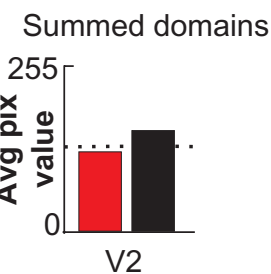

Figure 4 Diverse orientation correspondence in macaque area V2 (Case 4).

Notes: (A) Vessel pattern (left panel) and ocular dominance preference map (middle panel) indicating the functional VI/V2 border (yellow line). Color-coded real orientation map (right panel) indicates location of a "thin stripe" (dark band, orientation response is weak) and "pale/thick" stripes (color-coded band, orientation response is strong) in V2. Approximate location of thin versus pale/thick territories, marked above color-coded map. Scale bar I mm. (B) Magnified views of imaged area V2, indicating that thin stripe has complex (approximately rectangular) shape. Left panel; orientation response magnitude map, lightest areas indicate strongest orientation response. Right panel; color-coded response map indicating preferred orientation. (C) lllusory difference map; horizontal minus vertical illusory responses. Dark and light zones indicate preference for horizontal and vertical illusory contours respectively. Right panel; thresholded map, highlighting horizontal (red outline) and vertical (black outline) illusory preference domains. Grayscale: $-0.01 \%$ to $0.01 \% \mathrm{dR} / \mathrm{R}$. (D) Correspondence of real and illusory orientation preference maps. Left panel; color-coded vector map for real orientation. Right panel; grayscale difference map, horizontal minus vertical. Thresholded illusory orientation preference domains from (C) are superimposed in both maps. Some domains $(*)$ show "like-with-like" correspondence in V2 (eg, horizontal illusory associated with horizontal real domains). Other domains (+) show a "VI-like" correspondence in V2 (eg, horizontal illusory associated with vertical real). Grayscale: $-0.02 \%$ to $0.02 \% \mathrm{dR} / \mathrm{R}$. Scale bar I mm. (E) Split block analysis, V2 data only. Alternate data blocks were separately analyzed (aaa ... versus bbb ..., from original ababab ... data) to reveal consistent domain sign and locations. One domain in thin stripe is equivocal. (F) Real/illusory domain correspondence in V2 demonstrated by quantitative pixel analysis. Left panel; average (real) pixel values for each illusory domain shown in right panel of (C), indicating a diversity of domain relationships. Pixel values range from 0 (darkest) to 255 (lightest). Right panel; average (real) pixel values for all horizontal or vertical illusory domains. Pixel values range from 0 (darkest) to 255 (lightest). The relationship between real and illusory domains is less consistent in this case. Some horizontal (red bars) and some vertical illusory domains (black bars) show high pixel values, while horizontal illusory domains (red bars) show low real pixel values. Right panel; average (real) pixel values for all horizontal or vertical illusory domains shown. While there is a tendency for $\mathrm{V} 2$ vertical illusory domains (black bars) on average to show higher pixel values and horizontal illusory domains to show lower pixel values, this relationship is weak. (*) show "like-with-like" correspondence in V2; (+) show a "VI-like" correspondence in V2. Abbreviations: $d R / R$, \% change in reflectance; Avg, average; Pix, pixel; Horiz illu, horizontal illusory; Vert illu, vertical illusory. 

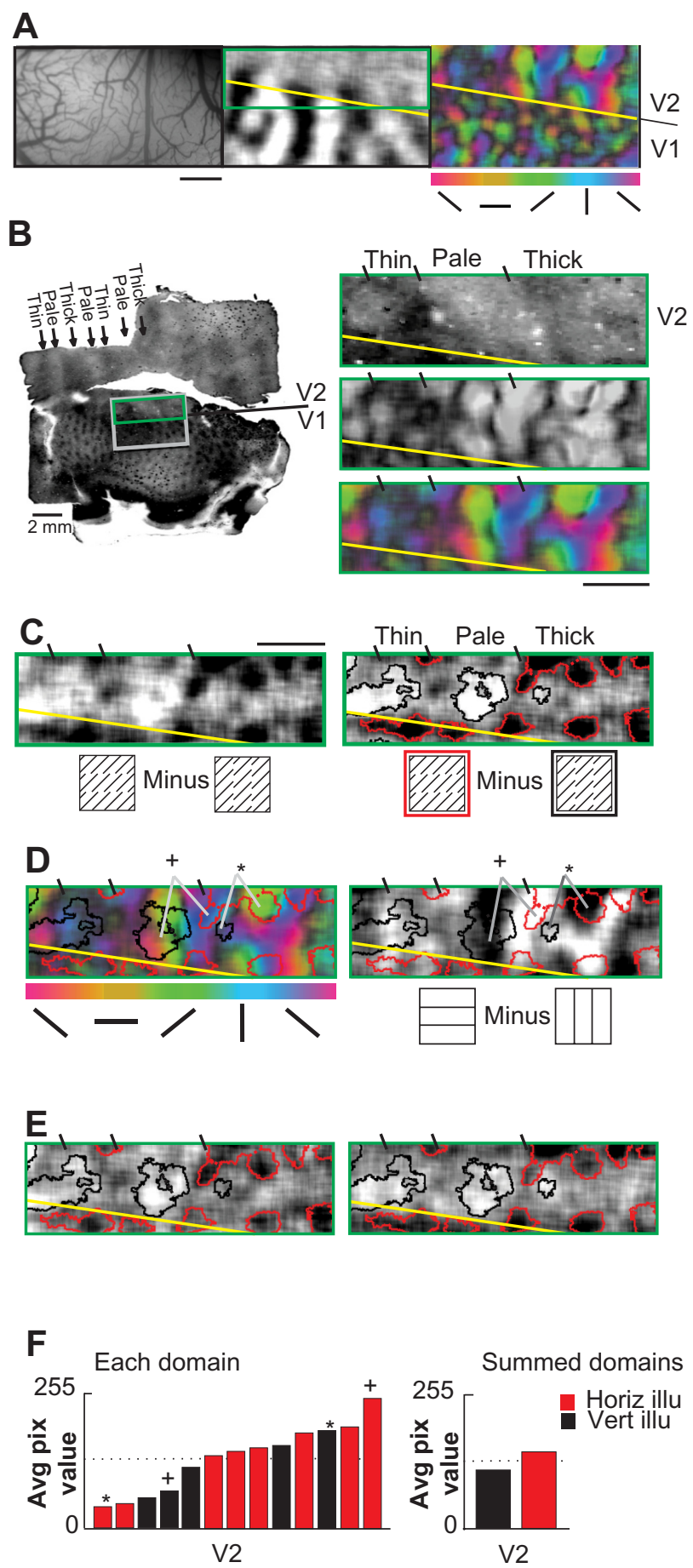

Figure 5 Diverse orientation correspondence in macaque area V2 (Case 5).

Notes: (A) Vessel pattern (left panel) and ocular dominance preference map (middle panel) indicating the functional VI/V2 border (yellow line). Color-coded real orientation map (right panel) indicates location of a "thin stripe" (dark band, orientation response is weak) and "pale/thick stripes" (color-coded band, orientation response is strong) in $\mathrm{V} 2$. Scale bar I mm. (B) Localization of V2 stripe compartments. Left panel; cytochrome oxidase histology for VI/V2 cortex including imaged regions (black rectangle for [A], green rectangle for [B-E]). Right panels; magnified views of area V2, indicating that thin stripe has complex (approximately rectangular) shape. Short black lines delineate approximate borders. Cytochrome oxidase histology (top right). Orientation response magnitude map (right middle); lightest areas indicate strongest orientation response. Color-coded response map (bottom right) indicating preferred orientation. (C) Illusory difference map V2; horizontal minus vertical illusory responses. Dark and light zones indicate preference for horizontal and vertical illusory contours, respectively. Right panel; thresholded map, highlighting horizontal (red outline) and vertical (black outline) illusory preference domains. Grayscale: $-0.01 \%$ to $0.01 \%$ dR/R. (D) Correspondence of real and illusory orientation preference maps. Left panel; color-coded vector map for real orientation. Right panel; grayscale difference map, horizontal minus vertical. Thresholded illusory orientation preference domains from (C) are superimposed in both maps. Some domains (*) show "like-with-like" correspondence in V2 (eg, horizontal illusory associated with horizontal real domains). Other domains (+) show a "VI-like" correspondence in V2 (eg, horizontal illusory associated with vertical real). Grayscale: $-0.02 \%$ to $0.02 \% \mathrm{dR} / \mathrm{R}$. Scale bar I mm. (E) Split block analysis, V2 data only. Alternate data blocks were separately analyzed (aaa ... versus bbb ..., from original ababab ... data) to reveal consistent domain sign and locations. (F) Diversity of real/illusory domain correspondence in $\mathrm{V} 2$ demonstrated by quantitative pixel analysis. Left panel; average (real) pixel values for each illusory domain shown in right panel of (C). Pixel values range from 0 (darkest) to 255 (lightest). Right panel; average (real) pixel values for all horizontal or vertical illusory domains. $\left(^{*}\right)$ show "like-with-like" correspondence in V2; (+) show a "VI-like" correspondence in V2. Abbreviations: dR/R, \% change in reflectance; Avg, average; Pix, pixel; Horiz illu, horizontal illusory; Vert illu, vertical illusory. 
in Ramsden et $\mathrm{al}^{23}$ ), there is evidence that neurons in V2 differentiate between illusory contours of different inducer composition $^{22}$ and that such differences in inducer content affect visual saliency. ${ }^{29}$ In Case 3 , there is a suggestion that illusory domains contain greater $45^{\circ}$ (green pixels) content than $135^{\circ}$ (red pixels) content, consistent with an inducerdependent bias in orientation representation. However, we have too few observations to make any conclusions regarding further specificity of real/illusory associations in V2.

We have thus directed our views of this issue to the discussion. In conclusion, illusory domains exhibited primarily coaligned real/illusory response, although in this case the association also included a broader range of orientation associations.

\section{Case 4}

In two of the five cases examined, we obtained further evidence of a complex spatial relationship between real and illusory orientation domains in area V2. Figure 4 presents imaging data obtained from the V1/V2 cortex of Case 4. The functional V1/V2 border was delineated (Figure 4A middle panel, large transverse surface blood vessel artefact, Figure 4A, left panel), revealing an approximately 1.5-mmwide strip of V2 on the surface. The locations of pale/thick V2 stripe compartments were determined by their strong orientation selectivity and the thin stripe locations by weak orientation selectivity (vector map is shown in Figure 4A, right panel). The two panels below (Figure 4B) show enlarged views of the V2 portion of this vector map. As shown by both the grayscale map (left) and the color-scale map (right), the region in the thin stripe exhibits primarily unoriented response. In this case, the "thin" stripe contains a weak orientation-selective domain (yellow). We would like to take this opportunity to reiterate a much observed but understated characteristic of V2 stripes. That is, while the thin/pale/ thick/pale organization is the most common description of V2 stripes, in practice the shape, size, and overall "solidity" of thin stripe compartments are more complex. . $^{611,30-34}$ Domains apparently associated with one stripe will invade a nearby stripe, and bridges and mergings between thin and thick stripes are observed. Thus, although we have indicated the locations of the V2 stripes (ticks above maps), these are based on traditional neuroanatomical conventions and should be interpreted loosely.

We then compared illusory contour response to this real orientation map. An illusory orientation difference map was obtained (unthresholded Figure 4C, left; thresholded Figure 4C, right), and demarcated illusory domains were overlayed on real orientation maps (Figure 4D). Consistent with the previous cases, the strongest illusory responses occurred in the thick/pale stripes. However, in this case we also observed two illusory domains in the defined thin stripe. Notably, the relatively weak horizontal illusory domain in this thin stripe (red outlined domain) coincided with horizontal real response (yellow domain) in this stripe (top left, Figure 4C), consistent with a like-to-like relationship. The other domain in this thin stripe (black outline) overlaid a non-oriented region (Figure 4A, lower panels) near the V1/ V2 border. Again, the apparent incidences of real/illusory orientation domains within the thin stripe may be associated with invasion of our designated thin stripe by the pale stripe compartment, and this should be borne in mind in our map comparisons.

In the pale/thick stripes, both "V1-like" (ie, anticorrelated, ie, real horizontal domains mapping onto illusory vertical domains, or real vertical domain mapping onto illusory horizontal domains) and "V2-like" (ie, like-withlike, ie, real horizontal domains mapping onto illusory horizontal domains, or real vertical domain mapping onto illusory vertical domains) alignments were observed. As in previous cases, V1 illusory orientation preference domains were commonly associated with orthogonal real orientation preference domains (Figure 4D, in V1 black outlines with yellow domains, red outlines with blue domains). In V2 of this case, however, we observed a mixed pattern of association. Some illusory and real domains in V2 exhibited a like-with-like spatial relationship (domains marked by an asterisk, Figure 4D). Other V2 domains appeared to exhibit a V1-like relationship. That is, V2 domains preferring horizontal or vertical illusory contours could sometimes prefer real orientations that were orthogonal to their illusory orientation preference. For example, the domains indicated by a cross in Figure 4D align in an orthogonal or V1-like correspondence (horizontal illusory domain [red outline] overlaps vertical real domain [blue shading]; vertical illusory domain [black outline] overlaps horizontal real domain [yellow shading], Figure 4D).

To verify that these V2 illusory maps were not simply spurious artefacts related to acquisition or analysis, we reexamined the data by splitting the collected data trials into two sets. ${ }^{35}$ Interleaving trial blocks were consecutively assigned to either set. New illusory difference maps were calculated for each set (Figure 4E, V2 data only). The overall pattern of illusory orientation preference was present in both maps, although one domain in the defined thin stripe (red outline) was equivocal across analyses. 
The mixed nature of real/illusory domain relationships was also evident in quantitative analyses (Figure 4F). For example, average (real map) pixel values for horizontal illusory domains marked by a cross in Figure 4D support the presence of V1-like real/illusory domain relationships in V2. Case 4 thus presented a mixed outcome, with domain alignments both V2-like (ie, like-with-like) and V1-like (ie, orthogonal) in correspondence. As with previous cases, some real orientation domains were not associated with either horizontal or vertical illusory activations.

\section{Case 5}

Evidence for diversity in illusory/real domain correspondence in V2 was also found in Case 5 (Figure 5). Again, we first mapped the V1/V2 border (Figure 5A, middle panel). We identified thin, pale/thick stripe compartments (Figure 5A, right panel) in V2 by mapping the magnitude of orientation selectivity. These compartment locations were confirmed via cytochrome oxidase histology (Figure 5B, left panel). Enlarged views of cytochrome oxidase histology (Figure 5B, upper right panel) and real orientation selectivity (Figure $5 \mathrm{~B}$, grayscale middle right panel, color-scale lower right panel) together indicate that a full thin/pale/thick cycle within V2 was imaged in this case. An illusory difference map was then obtained and thresholded to define illusory orientation preference domains (Figure 5C). Note that the strongest illusory responses (darkest and lightest pixels) occur in the thick/pale stripes. The correspondence between illusory and real orientation preference maps is shown in Figure 5D. As with Case 4, consistency of the illusory orientation map was confirmed by interleaved block split data analysis (Figure 5E).

This case revealed similar real/illusory domain relationships as Case 4. We observed V2 domains with a like-with-like real/illusory orientation preference correspondence (eg, domains marked by an asterisk, Figure 5D). However, we also observed V2 domains with an orthogonal real/illusory orientation preference correspondence (eg, domains marked by a cross, Figure 5D). Again, some real orientation response did not overlie either the horizontal or vertical illusory domains. These correspondences were evident in both pale and thick stripe compartments. Some illusory response was present at the margins of the thin stripes, though weaker than responses in pale/thick stripes (Figure 5C). Quantitative analysis of real map pixel values within demarcated illusory domains supported the presence of a range of domain relationships. These relationships included several instances of strong coalignments with both like-with-like and orthogonal real/illusory domain correspondence (Figure 5F).

\section{Discussion Illusory orientation preference is organized into domains in $\mathrm{V} 2$}

The aggregation of functional response (such as color, disparity, line orientation) into submillimeter-sized modules or domains is a fundamental organizational principle of early visual areas in primates. ${ }^{22}$ How higher-order features are represented in primate visual areas is less well understood. A clustering of illusory orientation preference has been suggested in area 18 of the $\mathrm{cat}^{36}$ and in the monkey. ${ }^{23}$ In the human cortex, some role for area V2 in the early representation of illusory contours has been suggested using other imaging methods (eg, functional magnetic resonance imaging and positron emission tomography), although these methods were unable to resolve activations at the scale of single functional domains. ${ }^{37-40}$

Here, we show that the principle of a submillimeter modular organization may also apply to representation of higherorder contour orientation in primate area V2. Our imaged data indicates that illusory orientation preference can cluster into distinct domains within V2. Similar to real orientation domains in V2, these domains are typically $0.25-0.75 \mathrm{~mm}$ in breadth, and range from approximately round to somewhat elliptical in shape. Because the design of the illusory stimulus constrains us to two orientations, we have not directly demonstrated that illusory contour response spans the range of orientations and thus were not able to generate a full illusory orientation map. However, we predict that such a full map exists and, given the observed size and spacing of V2 illusory orientation domains, would not be dissimilar in structure to that of the real orientation map. However, additional studies need to be conducted to address this.

\section{Illusory orientation preference domains are associated with thick/pale stripes}

Our data confirm the presence of V2 stripe organization and the association of thick and pale stripes with orientation selectivity. In particular, we confirm the parcellation of orientation representation in V2. This parcellation is in agreement with previous studies. ${ }^{23,27}$ Strongly activated and highly selective real orientation domains occurred predominantly within the thick and pale stripes while remaining sparse in thin stripes. Here, we have shown that a similar distribution of orientation exists for higher-order illusory orientation representation. Previous selected electrophysiological sampling of single units in V2 combined with neuroanatomical reconstructions suggested 


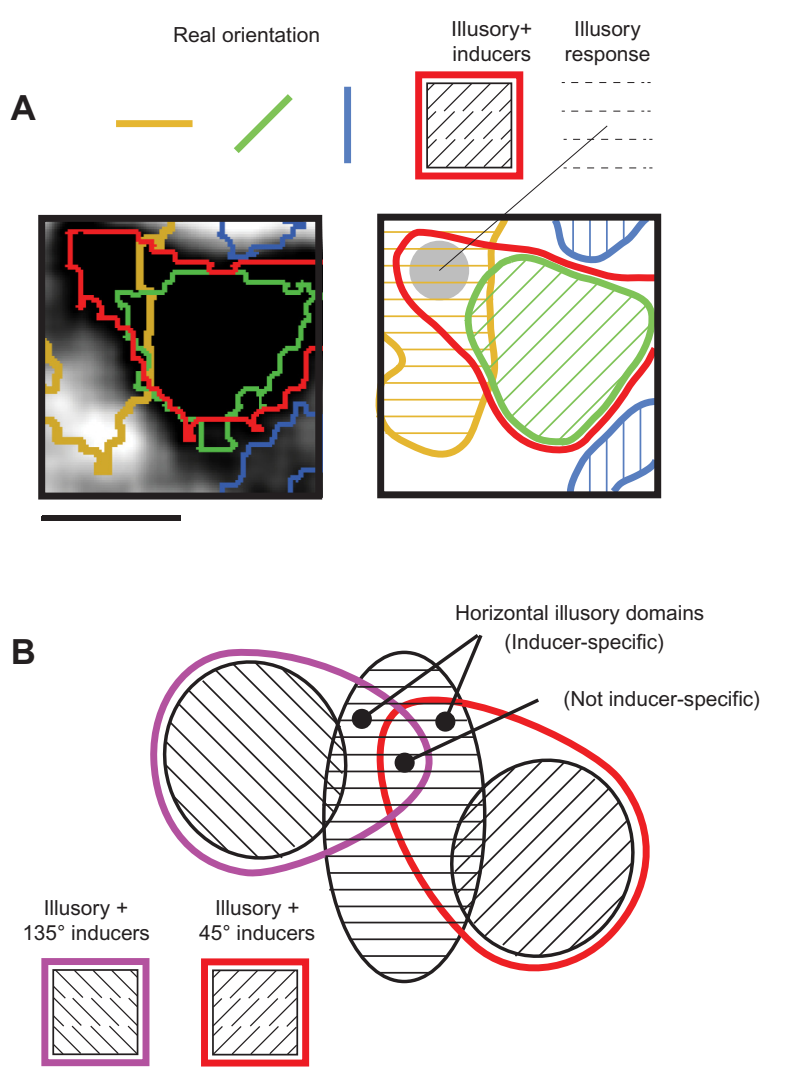

Figure 6 Putative schema for contour generalization in area V2.

Notes: (A) High magnification grayscale map of horizontal illusory response domain in V2. Scale bar 500 microns. Map is single-condition response (ie, relative to no stimulus, Figures I-5 difference or vector maps). Red outline; thresholded single-condition horizontal illusory domain. Other color outlines; overlaid real orientation domain locations (yellow, horizontal; green, $45^{\circ}$; blue, vertical). When horizontal illusory condition ( $45^{\circ}$ inducers) is presented, the $45^{\circ}$ orientation domain is activated (response to inducers). The activation extends into the horizontal domain (inferred contour activation) but avoids vertical domains. We suggest this context-dependent extension allows for a continuum of alignments of illusory and real orientation response. (B) We propose that V2 orientation domains include subregions with differing degrees of response to lower-order (real) and higherorder (illusory) features. Anticorrelated real/illusory alignments may represent exclusively real zones, exhibiting responses similar to orientation domains in VI (black outlined domain). Regions neither coaligned nor anticorrelated may represent the second tier illusory regions which extend into orientation domains of the inducer orientation (inducer-dependent zones, overlapping regions between red and black outlined domain and between purple and black outlined domain). Real/ illusory coalignments may represent "generalizing" or inducer-independent zones (overlapping region between black, red, and purple outlined domains).

a propensity for illusory responsive neurons within pale and thick stripes. ${ }^{41}$ Although we do not exclude the presence of illusory contour response from thin stripes, our case data indicate at least preferential clustering of oriented illusory contour activation in the thick and pale stripes, particularly when irregularity in thin stripe territory is taken into account. This study thus further confirms functional mapping evidence of preferential stripe location for illusory response organization in V2.

\section{A diversity of orientation response domains in $\mathrm{V} 2$}

We have previously described an organization for illusory response in V1 that is consistently anticorrelated with the real orientation response. ${ }^{23}$ Here, we report an organization for illusory response in V2 that is more complex than that in V1. In some cases, illusory response is predominantly correlated with matching real orientation response. This is consistent with the concept of a true higher-order orientation domain: ie, a domain sensitive to contour orientation regardless of the real or illusory nature of the stimulus. Unlike V1, however, this real/illusory domain relationship is not the only relationship evident. Indeed, our data suggest a range of associations between real and illusory orientation domains in V2. These include regions of anticorrelated or V1-like association, and regions of illusory response associated with real orientation domains that are neither aligned nor anticorrelated. Given the large number of trials summed, the similarity of maps from split block analyses, the consistent within-map anticorrelated real/illusory relationship in V1, and examples of impressive real/illusory alignments within $\mathrm{V} 2$, we have shown that this range of relationships is not artifactual. These data speak to the complexity of functional organization in V2, which suggests that the illusory response map is not simply a higher-order clone of the real orientation map in V2. Indeed, they suggest a realistic diversity in the interactions between real and illusory response in V2. This diversity is supported by data from single-unit physiology (see Consistency with single-unit electrophysiology section), and from studies on interactions between oriented cells in V1 and V2.32,42 Data from cross-correlation studies suggest the presence of multiple orientation networks between V1 and V2: a more tightly synchronized, orientation-preserving network (whose proposed function is to preserve local contour information) and a less synchronized orientation-diverse network (whose proposed function is to produce emergent higher-order transformations in V2) ${ }^{43}$ A plurality of orientation domains may be required to meet the diverse computations required within such networks for context-dependent contour feature detection. ${ }^{17}$

\section{Reliability of $\mathrm{V} 2$ illusory contour domain mappings}

The optical imaging method is an established tool for mapping real orientation domain organization. We applied identical methods to map illusory contour response in V2. ${ }^{9}$ Because electrophysiological studies report ${ }^{17}$ that as few as one-third of V2 cells may also respond to abutting grating illusory contours, imaged illusory response may be weaker than real response, or distributed into fewer domains. Illusory "signal" may thus be more difficult to detect within ongoing biological "noise". To address such possible confounds we averaged up to 140 response trials per mapping study, more than four 
times the number of trials commonly used for real orientation mapping. ${ }^{9,11}$ We are confident that our data are not simply noise because of the presence of distinct organizations at submillimeter scale, that were observed in separate experimental cases. We also revealed illusory/real mapping in V2 while simultaneously confirming previously reported anticorrelated real/illusory alignments in area $\mathrm{V} 1 .{ }^{23}$ We further established the reliability of domain mappings by performing additional split block analyses in two cases showing the most diversity in real/ illusory alignments. While our analyses may not necessarily detect relationships that exist between "active" domains that do not reach our threshold criteria, the strongest (and therefore most reliable) illusory domain responses can at least be demarcated and compared with real orientation maps.

\section{Consistency with single-unit electrophysiology}

Although previous studies ${ }^{18}$ emphasized that V2 cells may exhibit closely matched orientation preference for real and illusory stimuli, this may not necessarily be the only relationship existing in V2. Orientation preference of single $\mathrm{V} 2$ cells to real contours can differ from their preference to illusory contours. ${ }^{18,22}$ There may also be different classes of illusory contour cells, each encoding different levels of contour abstraction. ${ }^{18,22}$ For example, we have previously described three types of contour cell responses in V2: realonly, inducer-dependent illusory, and inducer-independent illusory. ${ }^{22}$ By this definition, real-only cells respond only to explicit or real line contours, inducer-dependent illusory cells are those tuned for illusory contours but only with specific inducer orientation, and inducer-independent illusory cells are those tuned for illusory contours regardless of inducer orientation. ${ }^{22}$ These different response types are consistent with the notion of a "generalizing" contour response, ${ }^{18}$ but indicate that the generalization may exist at varying degrees. Thus, although there may be a continuum of response profiles overall, we suggest a hierarchy of contour abstraction occurs in V2 at the cellular level. This diversity of generalization (and differing dependencies on specific induction components) in V2 cellular response may underlie the observed complexity of real/illusory associations expressed at the larger spatial scale of the functional domain.

\section{Contour generalization and relative real/illusory domain alignments in V2}

How might the degree of contour generalization in V2 relate to the particular real/illusory domain associations that we have described? Although speculative, we suggest a schema where the relative mappings of real/illusory orientation in V2 may predict the degree to which orientation domains will exhibit contour generalization. This schema offers explanation for the observed incomplete domain alignments: if an illusory contour domain is generalized (ie, independent of inducer orientation), real/illusory alignments are expected regardless of how the contour is inferred. However, if an illusory contour domain response is "conditional" (eg, depends on inference via $135^{\circ}$ but not $45^{\circ}$ inducer elements), then real/illusory alignment will only occur when those specific stimulus conditions are met.

We suggest that this dependency of illusory response on inducer orientation may evoke a bias in the location of an illusory domain - away from alignment with similar real (contour) orientation domain, and toward the real (inducer) orientation domain on which the activation depends. Figure 6A shows a high-magnification single-condition map obtained in response to horizontal illusory contour stimulus composed of $45^{\circ}$ inducers. Thresholded response domains are shown for real horizontal (yellow outlines), real $45^{\circ}$ (green outlines), and real vertical (blue outlines) domains. Note that the illusory horizontal domain (dark regions, thresholded in red) overlies the $45^{\circ}$ real domain (green). However it also invades the horizontal real (yellow) domains while avoiding the vertical real (blue) domains. Thus, in this single condition map, illusory stimulation activates real domains selective for the orientation of the illusory contour as well as real domains selective for the orientation of the inducers (Figures 1-5 "difference" maps where inducer orientations are subtracted out). That is, the inference of a higher-order (horizontal) contour by the arrangement of $\left(45^{\circ}\right)$ inducers may be associated with an apparent extension or "morphing" of the $45^{\circ}$ orientation response domain into the adjacent horizontal domain. This extension allows for a continuum of alignments of illusory contour response domain and the regular orientation map, and allows for zones within the contour response domain showing more sensitivity to a particular inducer orientation (inducer dependency).

We therefore propose that V2 orientation domains contain subregions with differing degrees of response to lower- and higher-order contour features (Figure 6B). Regions showing anticorrelated real/illusory alignments may be exclusively real zones (Figure 6B, black outlined domain), exhibiting a response similar to orientation domains in V1. Regions showing neither coaligned nor anticorrelated alignments may be the second tier (inducer-dependent) illusory regions which extend into orientation domains of the inducer orientation (Figure 6B, regions of overlap between red and black, 
and between purple and black outlined domains). Regions with real/illusory coalignments may be the "generalizing" or inducer-independent zones (Figure 6B, region of overlap between black, red, and purple outlined domains). Although speculative, this proposal puts forward a possible framework consistent with our observation that illusory contour domains bear a diverse range of relationships with real orientation response in $\mathrm{V} 2$.

\section{Experimental procedures Surgical preparation}

Experiments were performed under protocols approved by the Yale Institutional Animal Care and Use Committee. Four monkeys were anesthetized with thiopental sodium (1-2 mg/kg/hour intravenous [IV]), and one monkey (Case 5) was anesthetized with isoflurane $/ \mathrm{O}_{2}(0.8 \%-1.8 \%)$. To prevent involuntary eye position drift, all animals were paralyzed (vecuronium bromide, $100 \mu \mathrm{g} / \mathrm{kg} / \mathrm{hour}$ IV) and artificially respirated. Anesthetic depth was assessed continuously via implanted electroencephalography electrodes, end-tidal $\mathrm{CO}_{2}$, and heart rate monitoring, and by regular testing for response to toe pinch. Pupils were dilated with atropine drops and eyes were focused with primate contact lenses onto a computer graphics display. Under aseptic surgical conditions, a craniotomy and durotomy were performed to expose the cortex posterior to the lunate sulcus.

\section{Optical imaging}

An optical chamber (typically $2 \mathrm{~cm}$ in diameter) was adhered to the skull, filled with sterile silicone oil and sealed with a glass window. ${ }^{44-46}$ Images were acquired using an Imager 2001 system (Optical Imaging Inc., Rehovot, Israel; 630 nm light). Image data were binned to yield response map dimensions of $324 \times 240$ pixels. Each stimulus condition (Figure 1A) was presented in randomized order for 3 seconds ( $200 \mathrm{~ms}$ frames) with a $10-15$ second interstimulus interval. Signal-to-noise ratio was enhanced via trial averaging (40-140 trials per stimulus condition) and by synchronization of acquisition with heart rate and respiration. For ocular dominance mapping, computercontrolled electromechanical shutters were placed in front of the eyes. The camera was positioned with the lunate sulcus parallel to and just beyond the top edge of the field of view (Figure 1B, left panel), so as to record functional domains in exposed V2 and immediately adjacent area V1 (note: although the term functional domain has sometimes been associated with the notion of a fundamental cortical representational unit, here we use the term to mean a spatially concentrated functional preference for a particular stimulus feature).

\section{Visual stimuli}

Illusory contour and real line stimuli (Figure 1A) were created and presented binocularly to the animal using a custom-made C-language program. Real line stimuli were identically spaced (1.25 cycles/degree) and drifting line gratings (one pixel width, 0.8 degrees/second, drift range two cycles, 3-second presentation time, screen dimensions 13 [wide $\{\mathrm{w}\}] \times 10$ [high $\{\mathrm{h}\}$ ] degrees) presented binocularly at four orientations (horizontal $\left[0^{\circ}\right], 45^{\circ}$, vertical $\left[90^{\circ}\right]$, and $135^{\circ}$ ). In some instances, response to red/green isoluminant gratings were compared with response to achromatic gratings. Achromatic illusory contour gratings were composed of short $45^{\circ}$ lines (one pixel wide, 0.03 degrees width) spaced 0.25 degrees apart, a spacing which has been shown to be effective for illusory contour cells in V2. ${ }^{18,24}$ These inducing elements were aligned, with a column spacing of 1.25 cycles/degree, to produce a percept of either horizontal or vertical illusory contours. These rows of aligned inducers were together drifted back and forth (0.8 degrees/second, drift range two cycles, 3 -second presentation time, screen dimensions $13[\mathrm{w}] \times 10[\mathrm{~h}]$ degrees) in the direction along the orientation of inducing lines. This generated a percept of illusory contour motion orthogonal to the illusory contour orientation. Luminance values were measured using a calibrated photometer (Minolta Chroma Meter CS-100; Konica Minolta, Inc., Tokyo, Japan) and were constant across stimuli (background luminance $0.1 \mathrm{~cd} / \mathrm{m}^{2}$, line luminance $40.0 \mathrm{~cd} / \mathrm{m}^{2}$, global stimulus luminance $8.0 \mathrm{~cd} / \mathrm{m}^{2}$ ).

\section{Image analysis}

Analyses consisted of either difference maps or vector maps. Difference maps were determined by subtracting responses to one stimulus condition from responses to another stimulus condition. A grayscale preference map was then calculated indicating preference for one stimulus condition over the other. Vector maps were determined by first obtaining single-condition responses relative to a reference "cocktail blank" condition (sum of all presented conditions). ${ }^{47} \mathrm{~A}$ color-coded orientation preference map was then calculated by vectorially summing, pixel-by-pixel, the responses to all presented orientations (hue indicating net preferred orientation, and intensity of color indicating vector magnitude). ${ }^{7}$ Maps based on single-condition responses indicate all pixels responsive to the stimulus. Since single-condition illusory contour maps contain real orientation signal components, illusory specific responses are inferred by subtraction of two illusory contour conditions (eg, horizontal illusory minus vertical illusory); the 
common real line components are subtracted out, leaving the differential illusory contour response. ${ }^{36}$ All maps were calculated using first-frame subtraction procedures. ${ }^{47}$

To compare the locations of imaged illusory domains with the real orientation map, illusory contour difference maps were thresholded (eg, Figure 1F) at the 80th percentile to demarcate horizontal illusory domains (red outlines), and at the 20th percentile to demarcate vertical illusory domains (black outlines). ${ }^{23}$ Prior to thresholding, images were spatially filtered using a $9 \times 9$ pixel moving window low-pass filter. We presumed that if an activated group of pixels is too small, then it is less likely to be a true orientation domain and more likely to be due to noise. Thus, although infrequent, thresholded images (defined as groups of adjacent pixels) containing less than 10 pixels (ie, less than $50 \mu \mathrm{m}$ breadth) were excluded from analysis. Illusory and real orientation maps were qualitatively compared by overlaying thresholddefined illusory domains onto real orientation vector maps or difference maps (eg, Figure 1E). To quantitatively compare real and illusory maps, average pixel values (from real orientation preference maps) were calculated and graphically tabulated for demarcated illusory domains (eg, Figure 1F).

In Case 4 and Case 5, we verified a consistent map structure using a "split block" analysis method by recalculating two new maps from interleaving acquisition blocks associated with a single map. Data blocks that were acquired consecutively ("ababab") to determine the single map were reassigned to two new subsets ("aaa" and "bbb") and then each subset was analyzed independently and then displayed for qualitative comparison. We considered maps to be reliable signal rather than noise if the two new subset maps showed similar organizations and similar domain mapping relationships.

\section{Histology and alignment of anatomical and functional maps}

Following an overdose (100 mg pentothal IV), the animal was perfused transcardially with physiological saline followed by $4 \%$ paraformaldehyde. Imaged V1/V2 was removed, tissue samples were flattened, sunk in $30 \%$ sucrose, and then sliced $(30 \mu \mathrm{m})$ tangentially using a freezing microtome. Sections were processed using cytochrome oxidase histochemistry ${ }^{2}$ to anatomically identify the V1/V2 border and the stripe subcompartments of area V2. Functional images were first grossly aligned with the section based on small electrolytic lesions (7-10 $\mu \mathrm{A}$ for 10 seconds, using glass coated tungsten electrode tip in superficial cortical layers, depth $\sim 100-300 \mu \mathrm{m}$ ). They were then precisely aligned by comparing superficial vessel patterns recorded in vivo with Nissl-stained superficial layer histological sections (eg, Figure 1C). 12,35,48

\section{Disclosure}

The authors report no conflicts of interest in this work.

\section{References}

1. Tootell RB, Silverman MS, De Valois RL, Jacobs GH. Functional organization of the second cortical visual area in primates. Science. 1983;220(4598):737-739.

2. Wong-Riley MTT, Carroll EW. Quantitative light and electron microscopic analysis of cytochrome oxidase-rich zones in VII prestriate cortex of the squirrel monkey. J Comp Neurol. 1984;222(1):18-37.

3. deYoe, EA, van Essen DC. Segregation of efferent connections and receptive field properties in visual area V2 of the macaque. Nature. 1985;317:58-61.

4. Shipp S, Zeki S. Segregation of pathways leading from area V2 to areas V4 and V5 of macaque monkey visual cortex. Nature. 1985;315(6017): 322-325.

5. Hubel DH, Livingstone MS. Segregation of form, color, and stereopsis in primate area 18. J Neurosci. 1987;7(11):3378-3415.

6. Tootell RB, Hamilton SL. Functional anatomy of the second visual area (V2) in the macaque. J Neurosci. 1989;9(8):2620-2644.

7. Ts'o DY, Frostig RD, Lieke E, Grinvald A. Functional organization of primate visual cortex revealed by high resolution optical imaging. Science. 1990;249(4967):417-420.

8. Felleman DJ, Xiao Y, McClendon E. Modular organization of occipitotemporal pathways: cortical connections between visual area 4 and visual area 2 and posterior inferotemporal ventral area in macaque monkeys. J Neurosci. 1997;17(9):3185-3200.

9. Roe AW, Ts'o DY. Visual topography in primate V2: multiple representation across functional stripes. J Neurosci. 1995;15(5 Pt 2): 3689-3715.

10. Xiao Y, Zych A, Felleman DJ. Segregation and convergence of functionally defined V2 thin stripe and interstripe compartment projections to area V4 of macaques. Cereb Cortex. 1999;9(8):792-804.

11. Ts'o DY, Roe AW, Gilbert CD. A hierarchy of the functional organization for color, form and disparity in primate visual area V2. Vision Res. 2001;41(10-11):1333-1349.

12. Roe AW, Fristches K, Pettigrew JD. Optical imaging of functional organization in V1 and V2 of marmoset visual cortex. Anat Rec A Discov Mol Cell Evol Biol. 2005;287(2):1213-1225.

13. Levitt JB, Kiper DC, Movshon JA. Receptive fields and functional architecture of macaque V2. J Neurophysiol. 1994;71(6):2517-2542.

14. Yoshioka T, Dow BM. Color, orientation and cytochrome oxidase reactivity in areas V1, V2 and V4 of macaque monkey visual cortex. Behav Brain Res. 1996;76(1-2):71-88.

15. Malach R, Tootell RB, Malonek D. Relationship between orientation domains, cytochrome oxidase stripes, and intrinsic horizontal connections in squirrel monkey area V2. Cereb Cortex. 1994;4(2):151-165.

16. von der Heydt R, Peterhans E, Baumgartner G. Illusory contours and cortical neuron responses. Science. 1984;224(4654):1260-1262.

17. Peterhans E, von der Heydt R. Mechanisms of contour perception in monkey visual cortex. II. Contours bridging gaps. J Neurosci. 1989;9(5):1749-1763.

18. von der Heydt R, Peterhans E. Mechanisms of contour perception in monkey visual cortex. I. Lines of pattern discontinuity. J Neurosci. 1989;9(5):1731-1748.

19. Leventhal AG, Wang YC, Schmolesky MT, Zhou YF. Neural correlates of boundary perception. Vis Neurosci. 1998;15(6):1107-1118.

20. Bakin JS, Nakayama K, Gilbert CD. Visual responses in monkey areas $\mathrm{V} 1$ and V2 to three-dimensional surface configurations. J Neurosci. 2000;20(21):8188-8198.

21. Soriano M, Spillmann L, Bach M. The abutting grating illusion. Vision Res. 1996;36(1):109-116. 
22. Ramsden B, Hung C, Roe AW. Organization and circuitry of illusory contour domains in V1 and V2 of the macaque. Soc Neurosci Abstr. 1998;24:1506.

23. Ramsden BM, Hung CP, Roe AW. Real and illusory contour processing in area V1 of the primate: a cortical balancing act. Cereb Cortex. 2001;11(7):648-665.

24. Ramsden BM, Hung CP, Roe AW. Is there a substructure of functional organization for illusory contour processing in V2. J Vis. 2001;1(3):206.

25. Ramsden BM, Hung CP, Chen LM, Roe AW. Activation of illusory contour domains in Macaque Area V2 is accompanied by relative suppression of real contour domains in V1. Soc Neurosci Abstr. 1999;25:2060.

26. Ramsden BM, Chen LM, Hung CP, Roe AW. Sub-domain modulation of intrinsic optical imaging signals associated with illusory contour perception in area V2 of the macaque monkey cortex. Invest Ophthalmol Vis Sci. 1999;40(4):S369.

27. Pan Y, Chen M, Yin J, et al. Equivalent representation of real and illusory contours in macaque V4. J Neurosci. 2012;32(20):6760-6770.

28. Ramsden BM, Hung CP, Roe AW. Where does illusory response come from? Response of V1 cells in macaque monkey to local vs global stimulus attributes. Soc Neurosci Abstr. 2002;456:6.

29. Gillam B, Chan WM. Grouping has a negative effect on both subjective contours and perceived occlusion at T-junctions. Psychol Sci. 2002;13(3):279-283.

30. Horton JC. Cytochrome oxidase patches: a new cytoarchitectonic feature of monkey visual cortex. Philos Trans R Soc Lond B Biol Sci. 1984;304(1119):199-253.

31. Olavarria JF, Van Essen DC. The global pattern of cytochrome oxidase stripes in visual area V2 of the macaque monkey. Cereb Cortex. 1997;7(5):395-404.

32. Roe AW, Ts'o DY. The functional architecture of Area V2 in the Macaque monkey. In: Rockland KS, Peters A, Jones EG, Kaas JH, editors. Cerebral Cortex: Volume 12: Extrastriate Cortex in Primates. New York: Plenum Press; 1997:295-332.

33. Sincich LC, Horton JC. Pale cytochrome oxidase stripes in V2 receive the richest projection from macaque striate cortex. J Comp Neurol. 2002;447(1):18-33.

34. Vanduffel W, Tootell RB, Schoups AA, Orban GA. The organization of orientation selectivity throughout macaque visual cortex. Cereb Cortex. 2002;12(6):647-662.

35. Chen LM, Friedman RM, Ramsden BM, LaMotte RH, Roe AW. Fine-scale organization of primary somatosensory cortex (Area 3b) in the squirrel monkey revealed with intrinsic optical imaging. J Neurophysiol. 2001;86(6):3011-3029.
36. Sheth BR, Sharma J, Rao SC, Sur M. Orientation maps of subjective contours in visual cortex. Science. 1996;274(5295):2110-2115.

37. Hirsch J, DeLaPaz RL, Relkin NR, et al. Illusory contours activate specific regions in human visual cortex: evidence from functional magnetic resonance imaging. Proc Natl Acad Sci U S A. 1995;92(14): 6469-6473.

38. Ffytche DH, Zeki S. Brain activity related to the perception of illusory contours. Neuroimage. 1996;3(2):104-108.

39. Mendola JD, Dale AM, Fischl B, Liu AK, Tootell RB. The representation of illusory and real contours in human cortical visual areas revealed by functional magnetic resonance imaging. J Neurosci. 1999;19(19): 8560-8572.

40. Seghier M, Dojat M, Delon-Martin C, et al. Moving illusory contours activate primary visual cortex: an fMRI study. Cereb Cortex. 2000;10(7):663-670.

41. Peterhans E, von der Heydt R. Functional organization of area V2 in the alert macaque. Eur J Neurosci. 1993;5(5):509-524.

42. Nowak LG, Munk MH, James AC, Girard P, Bullier J. Cross-correlation study of the temporal interactions between areas V1 and V2 of the macaque monkey. J Neurophysiol. 1999;81(3):1057-1074.

43. Roe AW, Ts'o DY. Specificity of color connectivity between primate V1 and V2. J Neurophysiol. 1999;82(5):2719-2730.

44. Chen LM, Heider B, Williams GV, Healy FL, Ramsden BM, Roe AW. A chamber and artificial dura method for long-term optical imaging in primates. J Neurosci Methods. 2002;113(1):41-49.

45. Roe AW. Long-term optical imaging of intrinsic signals in anesthetized and awake monkeys. Appl Opt. 2007;46(10):1872-1880.

46. Ruiz O, Lustig B, Nassi JJ, et al. Optogenetics through windows on the brain in the nonhuman primate. J Neurophysiol. 2013;110(6): 1455-1467.

47. Bonhoeffer T, Grinvald A. Optical imaging based on intrinsic signals: The methodology. In: Toga AW, Mazziotta JC, editors. Brain Mapping: The Methods. New York: Academic Press; 1996:55-97.

48. Kaskan PM, Lu HD, Dillenburger BC, Kaas JH, Roe AW. The organization of orientation-selective, luminance-change and binocularpreference domains in the second (V2) and third (V3) visual areas of New World owl monkeys as revealed by intrinsic signal optical imaging. Cereb Cortex. 2009;19(6):1394-1407.
Eye and Brain

\section{Publish your work in this journal}

Eye and Brain is an international, peer-reviewed, open access journal focusing on clinical and experimental research in the field of neuro-ophthalmology. All aspects of patient care are addressed within the journal as well as basic research. Papers covering original research, basic science, clinical and epidemiological studies, reviews and

\section{Dovepress}

evaluations, guidelines, expert opinion and commentary, case reports and extended reports are welcome. The manuscript management system is completely online and includes a very quick and fair peer-review system, which is all easy to use. Visit http://www.dovepress.com/ testimonials.php to read real quotes from published authors. 